\title{
Yolk hormones and sexual conflict over parental investment in the pied flycatcher
}

\author{
Toni Laaksonen • Freya Adamczyk • Markus Ahola • \\ Erich Möstl • C(Kate). M. Lessells
}

Received: 24 May 2010 /Revised: 12 July 2010 / Accepted: 29 July 2010 / Published online: 13 August 2010

(C) Springer-Verlag 2010

\begin{abstract}
Female birds might be able to manipulate the parental effort of their male partner through elevated transfer of hormones to the eggs, since these hormones affect many chick traits that males might use as cues for adjusting the level of their investment. We experimentally studied whether female pied flycatchers Ficedula hypoleuca could manipulate male investment via yolk androgens. There is much more variation in yolk androgen levels between females than within clutches, and in order to change the androgen levels of the eggs, we swapped whole clutches between nests. To estimate the androgen levels of the clutch, we measured the androgen content of a single egg per clutch. Females did not succeed in manipulating male effort using yolk androgens, since there was no relationship between the division of parental care within a pair and either original or foster egg androgen levels. One of these relationships should have occurred if females were manipulating males. The proportion of feeding visits by the male was higher when the male was old (55\%) than when
\end{abstract}

Communicated by J. Graves

T. Laaksonen $(\bowtie)$

Finnish Museum of Natural History, University of Helsinki, FI-00014 Helsinki, Finland

e-mail: toni.k.laaksonen@helsinki.fi

T. Laaksonen $\cdot$ M. Ahola

Section of Ecology, Department of Biology, University of Turku,

Turku, Finland

F. Adamczyk $\cdot$ C. M. Lessells

Department of Animal Population Biology,

Netherlands Institute of Ecology (NIOO-KNAW),

Heteren, The Netherlands

E. Möstl

University of Veterinary Medicine,

Vienna, Austria he was young $(45 \%)$ and females laid eggs with higher androgen levels when mated with a young male. Young males did not exhibit any responses to yolk androgen levels either, which indicates that females cannot exploit their effort more than that of old males. We suggest that females may allocate yolk androgens to adjust the growth trajectories of the chicks to poor growing conditions when mated with young males that are poor providers or occupying a poor territory.

Keywords Aves · Birds · Compensatory investment . Differential allocation - Maternal effect · Parental care . Testosterone

\section{Introduction}

Male and female parents are in conflict over the investment they should make in their offspring, as each parent would benefit if the other made a higher share (Trivers 1972; Arnqvist and Rowe 2005; Houston et al. 2005; Lessells 2006). This sexual conflict arises from the fact that the parents are not related and that there is a trade-off between current parental investment and future reproductive success; a large investment in the current breeding attempt reduces the probability of surviving and reproducing successfully in the future (Williams 1966; Linden and Møller 1989; Lessells 1991). But how could one parent make the other work harder? One possibility would be to manipulate a cue that the partner uses for adjusting its level of care (Westneat and Sargent 1996). For example the feeding behaviour of parent birds is affected by several offspring traits, such as size (Slagsvold 1997; Lessells 2002), colour (Jourdie et al. 2004; de Ayala et al. 2007), and begging (Wright and Leonard 2002; Müller et al. 2007). Manipulation of any of 
these traits might thus provide a means of manipulating the other parent (Lessells 2006; Müller et al. 2007).

Recent studies have shown that maternally derived androgens in the eggs of birds affect several offspring traits, such as begging behaviour, early competitiveness and morphology (reviewed by Groothuis et al. 2005a; Gil 2008). It has therefore been suggested that female birds might be able to manipulate male effort to their benefit through elevated transfer of these hormones to the eggs (Lessells 2006; Moreno-Rueda 2007; Müller et al. 2007). A presumption for the manipulation to work is that elevating the androgen levels in the eggs should involve a cost or a constraint to the female, because otherwise all females would be expected to lay eggs with high androgen levels. This presumption appears to be fulfilled, as there is evidence for a cost of elevated androgen levels to the female herself (Clotfelter et al. 2004; Rutkowska et al. 2005) and to her chicks (Andersson et al. 2004; Groothuis et al. 2005b; Müller et al. 2005; Rubolini et al. 2007).

We conducted an experiment in which our primary aim was to examine whether female birds could manipulate male investment via yolk androgens. Our study species, the pied flycatcher Ficedula hypoleuca, is a good model for this purpose, because there is much variation in yolk androgen levels between females (Tobler et al. 2007). We designed the experiment using this fact and swapped whole clutches between nests, in order to change the androgen levels of the eggs in a nest in a natural way. In order to get an estimate of the androgen levels of the whole clutch, we measured the androgen content of a single egg per clutch. High repeatability of androgen levels within clutches (Tobler et al. 2007) indicates that the single egg gives a good estimate for the other eggs in the clutch.

There are two alternative ways on how male effort could be manipulated by females using yolk androgens: (1) the cue (offspring trait) used by parents to adjust their parental investment could be male-specific, i.e. only the male parent responds to changes in the cue; or (2) the cue could be general, i.e. the female also responds. In the latter case, females laying eggs with high androgen levels are expected to reduce their own responsiveness to the offspring so that only their males make a higher parental investment (Müller et al. 2007). These two mechanisms have different consequences for co-evolution of male and female strategies (Müller et al. 2007) and our second aim was therefore to determine which of them the females might use. We predicted that if females could manipulate males, one of the following two patterns should appear as shown in Fig. 1: (1) If females could manipulate males using a cue to which only the males respond, the proportional investment by the male should increase with increasing androgen level in the foster eggs. (2) Alternatively, if females could manipulate male investment using a cue to which both sex parents respond, the proportional investment

\section{Predictions when the cue is:}

a) male-specific:

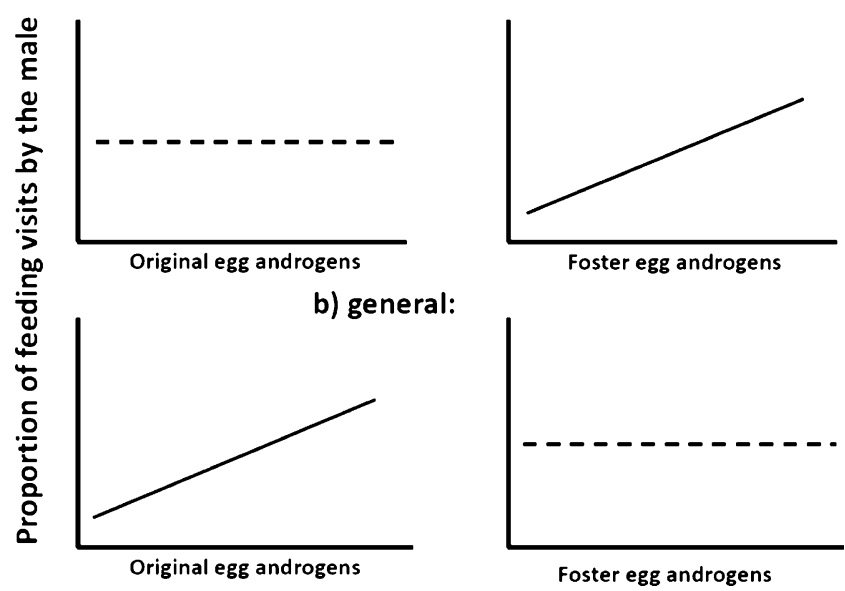

Fig. 1 The predictions for the experiment. a If females can manipulate male investment using a cue to which only males respond, proportional investment by the male should increase with increasing androgen level in the foster eggs. There is no clear prediction for the relationship between proportional investment by the male and the original egg androgen levels. b If females can manipulate male investment using a cue to which both sexes respond, the proportional investment by the male should increase with increasing androgen level in the original eggs of the nest. This is because a female that lays eggs with high androgen levels should reduce her responsiveness to the androgen-mediated cue(s) of the chicks. There is no clear prediction for the relationship between the proportional investment by the male and foster egg androgen levels

by the male should increase with increasing androgen level in the original eggs of the nest. This is because a female that lays eggs with high androgen levels should have low responsiveness to the androgen-mediated cue(s) of the chicks (in other words, she should be more insensitive to the cue that would otherwise make her feed more).

Manipulation of male investment should always benefit the female, but it has been proposed that the attempt to manipulate the male could also be condition-dependent. For example, females could try to manipulate male effort when they are mated with a male that is a poor-quality provider (e.g. an inexperienced young male), in order to make the male compensate for his poor parental skills with higher parental effort (Michl et al. 2005; Navara et al. 2006). Females could also do this when mated with an attractive male, to keep him involved in parental care and in this way prevent him from searching for other mating opportunities (Groothuis et al. 2005a). This could explain why females in many species lay eggs with high androgen levels when they are mated to an attractive male (Groothuis et al. 2005a; Moreno-Rueda 2007). We therefore studied whether the potential manipulation by the female could depend on male traits, such as age or sexual ornamentation. To this end, we first examined whether male traits affect the yolk androgen deposition by the female. 


\section{Material and methods}

Study population, study design and general methods

The study was conducted in a nest box population of pied flycatchers on the island of Ruissalo in Turku, Finland (ca. $60^{\circ}$ $25^{\prime} 60 \mathrm{~N} 22^{\circ} 10^{\prime} 0 \mathrm{E}$ ), in summer 2005 . The pied flycatcher is a migratory species that arrives at the breeding grounds in May. The nest boxes were routinely monitored every third day from early in May for nest initiation and egg-laying. Pied flycatchers lay one egg per day and we could thus detect each nest before the fourth egg was laid. Nests were visited on the day when the third egg was laid and the three eggs in the nest were marked with small dots using a non-toxic permanent marker. On the following day, the fourth (unmarked) egg was collected and replaced with a dummy egg (to maintain the same incubation effort). The collected egg was weighed to the nearest $0.01 \mathrm{~g}$ with a digital balance and then frozen at $-20^{\circ} \mathrm{C}$ until analyses. The concentrations of immunoreactive androstenedione (A4) and testosterone (T) in the yolks were measured later in the lab with enzyme immunoassays, following the methods described in Hackl et al. (2003). Based on 24 clutches collected from the study population in 2004, the androgen levels in the fourth egg are strongly correlated with the clutch mean (Spearman rank correlation coefficient, $r_{\mathrm{s}}=0.89$ for $\mathrm{T}$ and $r_{\mathrm{s}}=0.80$ for A4).

In the middle of the incubation period ( 5 days after clutch completion), we swapped whole clutches between two or three nests with the same laying date. The modal clutch size in the study population is six eggs and we only used clutches of six or seven eggs (including the collected egg). The eggs were transported between nests in under $10 \mathrm{~min}$ in a box with a separate cotton-softened compartment for each egg. The removed eggs were temporarily replaced with dummy eggs in the nest from which the swapping was begun. In most cases the female had already returned to incubate the (dummy) eggs when the foster clutch was taken to the nest.

Nests were visited daily from the estimated hatching (after 14 days of incubation), to determine the actual hatching date. One or two days after hatching the brood size was standardised to six chicks. In clutches of six eggs, this meant that the dummy egg was replaced with a chick from a reserve nest. In clutches of seven eggs, one of the six chicks was swapped with a chick from another nest. This way, each experimental nest had six chicks, one of which was from a different brood. Most of the replacement chicks were taken from nests from which an egg had also been collected, but in some cases we had to take a chick from an extra nest outside the main study area because the replacement chick needed to fit within the age range of the other chicks in the nest ( $0-2$ days). In a few cases one of the eggs did not hatch and two replacement chicks were brought to the nest. The nest was checked again 4 days after hatching. If one of the chicks had died, it was replaced with another chick from another nest, in order to maintain the brood size of six chicks for the measurement of parental feeding rates the next day. The small chicks were marked family-wise by clipping downy feathers either from the feather tufts on the head or back (only to an extent allowing recognition). The feathers that were cut are dead tissue (like hair), so this did not cause any pain or loss of blood to the chicks. The yolk androgen levels for a foster brood were estimated as the mean level in the single eggs collected from clutches from which the chicks originated, weighted by the number of chicks from each original clutch. The sample size for measurements of parental feeding rates was 50 nests. In these nests there were nine chicks (from a total of 300) for which we had no estimate of yolk androgen levels in their nest of origin. These nine chicks were distributed in nine different nests, in which there were always five other chicks for which we had the estimate. We consider it thus very unlikely that this low proportion (3\%) of chicks with no estimate of yolk androgen levels would have had any meaningful diluting effect on our results.

\section{Measurement of feeding rates}

Parental feeding rates were recorded for $2 \mathrm{~h}, 5$ days after hatching. We chose to record parental care at 5 days as a compromise between the chicks having reached a size where they make substantial demands on the parents, and the likely waning influence of maternally derived yolk androgens relative to that of androgens produced by the chick itself. A camouflaged video camera was placed 10-20 m from the nest. This was done without visiting the nest, in order to avoid disturbance. After video-recording, the chicks were marked with individually coded aluminium rings, and their wing length and mass measured. The video-recording yielded 44 recordings of $120 \mathrm{~min}$ and six recordings of 67-113 $\mathrm{min}$. We therefore expressed feeding rates for each parent as visits per hour.

\section{Measurement of the parents}

Female parents were captured at the end of the incubation period either by hand or using a swing-door trap when they returned to incubate. The age of the female (1-year old or older) was determined on the basis of feather wear (Lundberg and Alatalo 1992; Svensson 1992). Male parents were captured with a swing-door trap 6-7 days after the chicks hatched. The following male traits were measured: age (oneyear old or older), tarsus length, wing length, dorsal coloration, forehead patch size and feather UV reflectance. Dorsal coloration of pied flycatcher males ranges continuously from fully black to fully brown, with most of the males in this population being either intermediate or predominantly 
black. The dorsal coloration was measured with a traditional Drost score (Drost 1936; Lundberg and Alatalo 1992), which ranges from I (fully black head and back) to VII (fully brown head and back). Although the score is a classification, the underlying trait is continuous and we therefore used it as a continuous variable in the analyses. The forehead patch of male pied flycatchers is another highly variable feature of the breeding plumage (Lundberg and Alatalo 1992). Forehead patch size was measured from digital photographs taken in the field with a millimetre grid held next to, and at the same level and angle as, the patch. The patch area was measured using manual selection of the patch in Image-J freeware. Female pied flycatchers choose their mate on the basis of the brightness of male coloration at ultraviolet (UV) wavelengths (Siitari et al. 2002) and this trait also affects the probability of the male losing paternity in his nest (Lehtonen et al. 2009). UV coloration was measured from the central tertial feather, which was collected from each individual. This feather is in the centre of the white wing patch, which is much larger in the breeding plumage of males than females. We measured UV coloration from this feather, because white feathers typically have higher UV reflectance than dark feathers (Eaton and Lanyon 2003) and the middle tertial has a rigid structure whereas the dark or brown body feathers on the back have a soft semi-plume structure. The UV chroma [proportion of reflectance in the UV spectrum, i.e. (reflectance at $320-400 \mathrm{~nm}) /($ reflectance at $320-700 \mathrm{~nm})]$ was measured in the lab with a spectrophotometer (Avantes 2048, Avantes DH-S light source; white standard WS-2).

The experiment was conducted under the licences LOS2004-L-349-254 from the Southwest Finland Regional Environment Centre and 1524/05 from the Lab-Animal Care and Use Committee of the University of Turku.

\section{Statistical analyses}

The analyses were conducted with generalised linear models in SAS 9.1. The proportion of feeding visits by the male was analysed using the number of male feeding visits as the response and total number of feeding visits (by both parents) as the denominator (Proc GENMOD, events/trials syntax, logit link, binomial distribution). We carried out the analyses on the proportion of feeds by the male, but this is simply a measure of the relative investment by males and females, and the results of the statistical analyses would be identical if the data were expressed as proportion of feeds by the female. Other response variables were normally distributed and analysed with Proc GLM (using type III sums of squares). The models were simplified by backward elimination of non-significant variables $(\alpha=0.05)$. The non-significance of each removed variable was confirmed by adding the variable alone to the final model. Model residuals were routinely checked for normality, heteroschedasticity and non-linear patterns.
The concentrations of the two hormones (A4 and T) were positively correlated $(r=0.58, P<0.0001, N=95)$ and thus could not be considered independent from each other in the analyses. We therefore performed a principal component analysis on them and used the first principal component (PC1) as a measure of androgen concentration in the analyses. PC1 captured $79 \%$ of the total variation and was highly correlated with the concentrations of both hormones (A4: $r=0.89$, $P<0.0001, N=95 ; \mathrm{T}: r=0.89, P<0.0001, N=95)$. Increasing values of $\mathrm{PC} 1$ indicate increasing values of both hormones. Although we have based our interpretations solely on PC1 in this paper, we have also given details of the results for $\mathrm{A} 4$ and $\mathrm{T}$ separately (Fig. 2). We have done this in order to make these data useful and available to future researchers.

\section{Results}

Yolk androgen concentrations in relation to male and female traits

Yolk androgen level was not associated with the plumage traits of males (Table 1), but females that were mated to 1year-old males laid eggs with higher androgen concentration than those mated to older males (Table 1, Fig. 2). This indicates that a potential attempt by the female to manipulate male effort through yolk androgens does not depend on male attractiveness (as measured by UV reflectance, forehead patch size or dorsal coloration). However, it suggests that it could depend on male age, with females mated to young males attempting to make their mates compensate for their potentially lower parenting skills or parental effort. Yolk

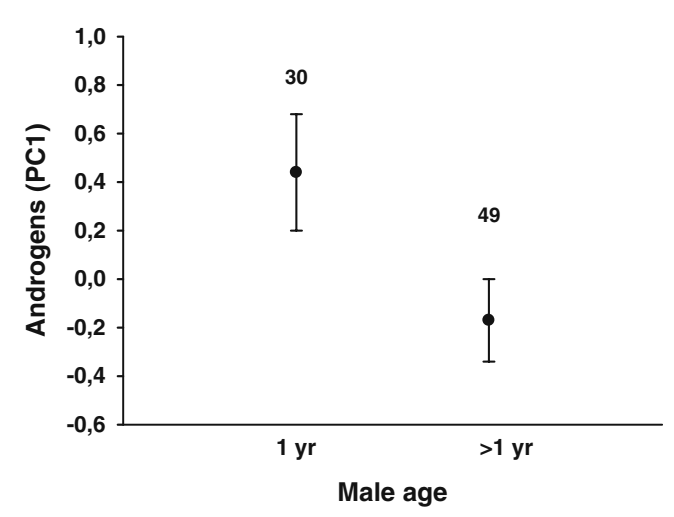

Fig. 2 Difference in the androgen levels (PC 1) in the eggs laid by partners of 1-year-old and older male pied flycatchers (mean \pm s.e.). Sample size (one egg (the fourth) per clutch) is indicated above the bars. The corresponding mean $( \pm$ s.e.) concentrations of the two hormones separately were: $124.89 \pm 12.44 \mathrm{ng} / \mathrm{g} \mathrm{A} 4$ and $15.76 \pm$ $1.23 \mathrm{ng} / \mathrm{gT}$ for 1-year-old males; and $98.28 \pm 6.03 \mathrm{ng} / \mathrm{g} \mathrm{A} 4$ and $13.08 \pm 0.83 \mathrm{ng} / \mathrm{gT}$ for older males $\left(\mathrm{F}_{1,77}=3.47, p=0.067\right.$ for $\mathrm{A} 4$, and $\mathrm{F}_{1,77}=3.49, p=0.066$ for $\mathrm{T}$ ) 
Table 1 The relationships of male traits, female age and laying date with yolk androgen concentration ( $\mathrm{PC} 1$, based on yolk testosterone and androstenedione levels)

\begin{tabular}{llll}
\hline & DF & $F$ & $P$ \\
\hline $\begin{array}{l}\text { Reduced model } \\
\quad \text { Male age }\end{array}$ & 1,77 & 4.42 & 0.039 \\
$\begin{array}{l}\text { Removed variables } \\
\text { Drost score }\end{array}$ & 1,75 & 0.24 & 0.62 \\
Forehead patch size & 1,73 & 0.31 & 0.58 \\
UV chroma of wing patch & 1,71 & 0.05 & 0.83 \\
Female age & 1,75 & 0.00 & 0.96 \\
Laying date & 1,76 & 0.23 & 0.63 \\
\hline
\end{tabular}

The statistics for the removed variables are from a model in which they were added alone to the final reduced model

The error DF varies due to missing data for some of the variables $D F$ degrees of freedom; $F$ F-statistic

androgen concentration was not associated with female age or timing of breeding (1st egg-laying date; Table 1).

Feeding rates

The proportion of feeding visits by the male was not related to the yolk androgen levels of either the foster or original clutch (Table 2; Fig. 3). This indicates that females did not manipulate male investment via yolk androgens. The proportion of feeding visits by the male was, however, strongly influenced by male age: old males made on average $55 \%$, and 1 -year-old males only $45 \%$, of feeding visits by the pair (Table 2; Fig. 4a). This was primarily due to females mated to 1 -year-old males making more feeding visits than those mated to older males $\left(F_{1,48}=21.20, P<\right.$

Table 2 Results of the Generalized Linear Models (logit link, binomial distribution) explaining the proportion of feeding visits by the male (number of visits by male/total number of feeding visits by the pair)

\begin{tabular}{lccc}
\hline & DF & $\chi^{2}$ & $P$ \\
\hline $\begin{array}{l}\text { Reduced model } \\
\quad \text { Male age }\end{array}$ & 1,48 & 10.88 & 0.001 \\
$\begin{array}{l}\text { Removed variables } \\
\text { Androgens foster eggs (PC1) }\end{array}$ & 1,47 & 0.02 & 0.89 \\
Androgens original eggs (PC1) & 1,47 & 0.18 & 0.69 \\
Foster*original egg androgens & 1,45 & 0.03 & 0.87 \\
Male age*androgens foster eggs & 1,46 & 1.19 & 0.28 \\
Male age*androgens original eggs & 1,46 & 0.11 & 0.73 \\
Female age & 1,47 & 1.88 & 0.17 \\
Laying date & 1,47 & 0.70 & 0.40 \\
\hline
\end{tabular}

$D F$ degrees of freedom; $\chi^{2}$ chi-square statistic
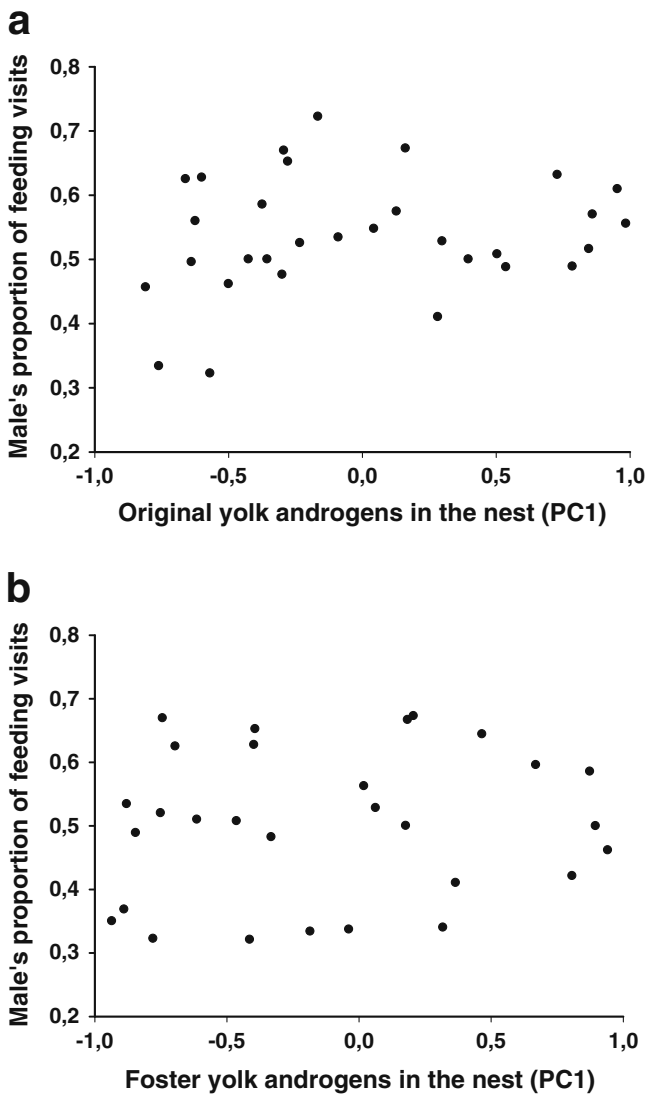

Fig. 3 The proportion of feeding visits by the male in relation to (a) original yolk androgen levels in the nest (PC 1), and (b) foster yolk androgen levels in the nest (PC1)

0.0001 , Fig. 4b). The difference in the feeding rates between 1-year-old and older males was not significant $\left(F_{1,48}=0.94, P=0.33\right.$, Fig. 4c). There was no obvious difference in feeding rate between 1-year-old and older females $\left(F_{1,48}=1.87, P=0.18\right.$, mean \pm s.d.: 1-year-old: $13.89 \pm 0.50, N=10$; older: $12.25 \pm 1.32, N=40)$. The total number of feeding visits per hour by the pair members was higher when the male was young $\left(F_{1,48}=5.47, P=0.02\right.$; mean \pm s.d.: 1-year-old: 28.07 \pm 4.35 ; old: $25.20 \pm 4.13$ ). There were no significant interactions between original and foster yolk androgen levels or between either of these and male age (Table 2). The absolute feeding frequencies of neither male nor female parents were related to original or foster yolk androgen levels (all $P>0.5$ ).

\section{Discussion}

Yolk androgens and sexual conflict

Our results indicate that female pied flycatchers do not succeed in manipulating male effort using yolk androgens, since there was no relationship between the division of 


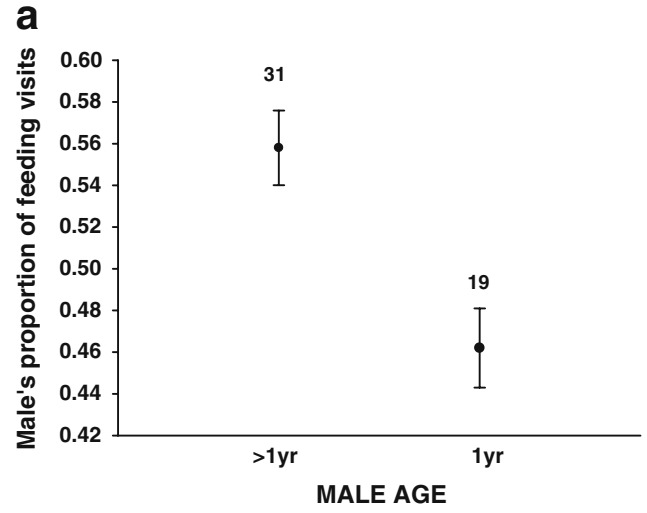

b

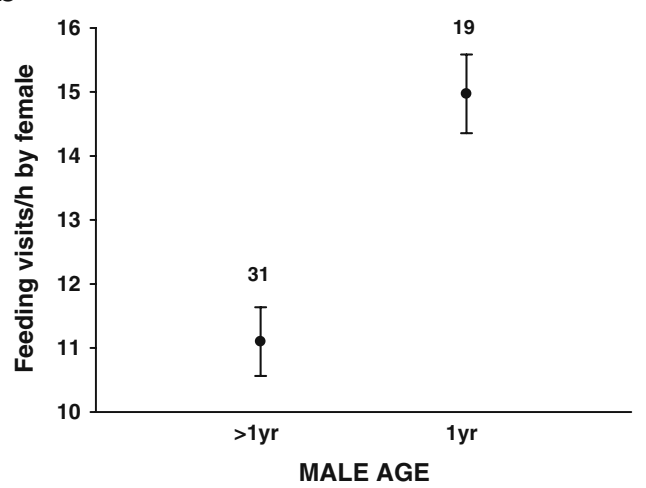

C

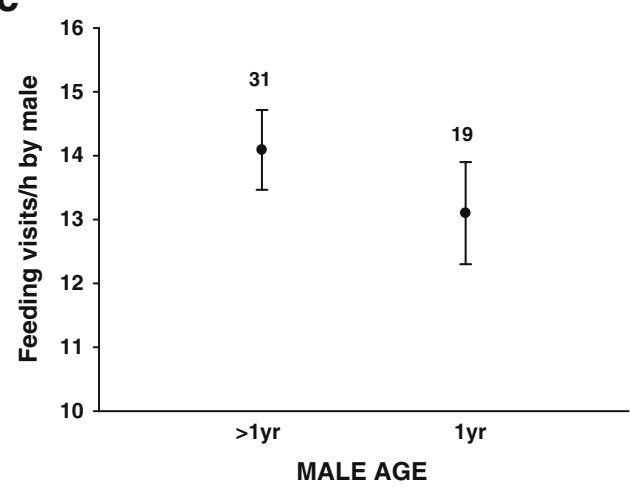

Fig. 4 (a) The proportion of feeding visits made by the male in relation to male age (1-year-old or older). (b) Feeding visits/hour of the female when mated with 1-year-old or older males. (c) Feeding visits/hour by 1-year-old and older males. Means \pm s.e. are shown with sample size above the bars

parental care within a pair and either original or foster egg androgen levels. One of these relationships should have occurred if females were manipulating males. Our results also do not support the idea of condition-dependent manipulation (that would depend on male traits); although we found that females which mated with 1-year-old males laid eggs with higher androgen levels than those mated with older males, the young males did not exhibit any obvious responses to the yolk androgen levels in their foster clutch.
These results thus add to the evidence that female birds do not manipulate male investment through yolk androgens (Tschirren and Richner 2008; Ruuskanen et al. 2009), despite suggestions that this might be adaptive (Groothuis et al. 2005a; Michl et al. 2005; Lessells 2006; Moreno-Rueda 2007; Müller et al. 2007). It therefore seems that sexual conflict has less influence on yolk androgen allocation than factors such as social density, parental quality or food availability (reviewed by Groothuis et al. 2005a, Gil 2008).

We designed our experiment on the basis of greater variation in androgen levels among than those within nests, and swapped whole clutches, in order to change the androgen levels of the eggs in a nest. Another approach would have been to directly manipulate the hormone levels of the eggs (Groothuis et al. 2005a). Our design has both disadvantages and advantages: the main disadvantage of our procedure is that it also changes the levels of other components that co-vary with the androgen levels, so an apparent effect of yolk androgens could be due to another egg component. For example, in the collared flycatcher Ficedula albicollis, a cross-fostering experiment similar to ours indicated that yolk androgens might mediate sexual conflict over parental investment, but the effect cannot be unequivocally attributed to yolk androgen levels rather than some co-varying egg component (Ruuskanen et al. 2009). However, a concomitant benefit of our design is that we can conclude that none of the egg components co-varying with androgen levels mediates sexual conflict over parental care in the pied flycatcher. Another advantage of our design is that it maintains natural co-variation between hormones and other egg constituents, thus avoiding responses due to unnatural egg composition. Yet some caution on the generality of these results needs to be taken because the responses of the parents might depend on conditions and thus differ between years or environments. The results could also vary according to the age of the nestlings, which could not be considered with the current data. It is furthermore not known to what extent and which nestling traits are affected by yolk androgens in the pied flycatcher, although it is known that they have sex-specific long-term effects on behaviour (Ruuskanen and Laaksonen 2010).

Yolk androgens in relation to male age

Although we found no evidence that females could manipulate the effort of young males, one possible explanation for the observed pattern is that females evolved to exploit young males but the males then evolved to neglect the cues that females could use for exploitation. It can indeed be expected that in many cases co-evolution will quickly lead to an endpoint where the manipulation is no longer adaptive. There are, however, other potential explanations for the higher androgens levels in clutches of 
young males that we consider at least as likely. First, it may be that young males have poorer-quality territories or that they provide less courtship feeding than older males, which directly affects the condition of the female during egglaying. This view is supported by the evidence that female pied flycatchers in better condition laid eggs with lower androgen levels than those in poor condition (Tobler et al. 2007), which has also been found in experimental food supplementation studies on other species (Verboven et al. 2003; Gasparini et al. 2007; but see Warner et al. 2007). Second, it may be that females somehow perceive the young male or his territory as poor future growth conditions for the nestlings, and adjust the growth strategy of the nestlings to these conditions via yolk androgens. This could be supported by the finding that the feeding rate of females themselves was higher when the male was young, which could indicate that they were also compensating for poor provisioning by the young male; although there was no difference in the feeding rates between young and old males, the young males may have been delivering smaller prey loads than the older ones. High yolk androgen levels might be beneficial in poor growth conditions because they have, for example, been shown to improve the survival of nestlings in harsh conditions, which may have been due to more efficient energy use (Pilz et al. 2004). Yet a third possibility is that the females compensate via yolk androgens for the potentially lower genetic quality of the offspring of young males (older males may be on average of higher quality because they have gone through a longer period of selection; Hegyi et al. 2006). Distinguishing between these alternatives requires further experiments, in which it is important to consider that the costs or benefits of yolk androgens may also arise through long-term effects on the phenotype (e.g. Ruuskanen and Laaksonen 2010).

\section{Conclusions}

The suggestion that female birds could manipulate male investment through yolk androgens has been made several times (Groothuis et al. 2005a; Lessells 2006; Michl et al. 2005; Moreno-Rueda 2007; Müller et al. 2007), but as yet there is no evidence that this occurs (this study; Tschirren and Richner 2008; Ruuskanen et al. 2009). It therefore seems that sexual conflict does not play a significant role in yolk androgen allocation but rather that females may allocate yolk androgens to adjust the growth trajectories of the chicks to poor growing conditions when for example mated with young males that are poor-quality providers or occupying a poor quality territory. In more general terms, this may be seen as an example of compensatory investment/negative differential allocation (e.g. Bolund et al. 2009; Ratikainen and Kokko 2010). A key question to be solved next is whether laying eggs with higher androgen levels when mated with young males is a plastic and adaptive response by the female to male age and/or the resources associated with it (Marshall and Uller 2007; Lessells 2008).

Acknowledgements We thank Jorma Nurmi and Esa Lehikoinen for help and advice in field work, Suvi Ruuskanen for comments on the manuscript, and Academy of Finland and Emil Aaltonen Foundation for funding (grants to TL).

\section{References}

Andersson S, Uller T, Lõhmus M, Sundström F (2004) Effects of egg yolk testosterone on growth and immunity in a precocial bird. J Evol Biol 17:501-505

Arnqvist G, Rowe L (2005) Sexual conflict. Princeton University Press, Princeton and Oxford

Bolund E, Schielzeth H, Forstmeier W (2009) Compensatory investment in zebra finches: females lay larger eggs when paired to sexually unattractive males. Proc Roy Soc Lond B 276:707715

Clotfelter ED, O'Neal DM, Gaudioso JM, Casto JM, Parker-Renga IM, Snajdr EA, Duffy DL, Nolan J, Ketterson ED (2004) Consequences of elevating plasma testosterone in females of a socially monogamous songbird: evidence of constraints on male evolution? Horm Behav 46:171-178

de Ayala RM, Saino N, Møller AP, Anselmi C (2007) Mouth coloration of nestlings covaries with offspring quality and influences parental feeding behavior. Behav Ecol 18:526-534

Drost R (1936) Über das brutkleid männlicher Trauerfliegenfänger, Muscicapa hypoleuca. Vogelzug 6:179-186

Eaton MD, Lanyon SM (2003) The ubiquity of avian ultraviolet plumage reflectance. Proc Roy Soc Lond B 270:1721-1726

Gasparini J, Boulinier T, Gill VA, Gil D, Hatch SA, Roulin A (2007) Food availability affects maternal transfer of androgens and antibodies into the eggs of a colonial seabird. J Evol Biol $20: 874-880$

Gil D (2008) Hormones in avian eggs: physiology, ecology and behavior. Adv Study Behav 38:337-398

Groothuis TGG, Müller W, von Engelhardt N, Carere C, Eising C (2005a) Maternal hormones as a tool to adjust offspring phenotype in avian species. Neurosci Biobehav Rev 29:329352

Groothuis TGG, Eising CM, Dijkstra C, Müller W (2005b) Balancing between costs and benefits of maternal hormone deposition in avian eggs. Biol Lett 1:78-81

Hackl R, Bromundt V, Daisley J, Kotrschal K, Möstl E (2003) Distribution and origin of steroid hormones in the yolk of Japanese quail eggs (Coturnix coturnix japonica). J Comp Physiol B 173:327-331. doi:10.1007/s00360-003-0339-7

Hegyi G, Rosivall B, Török J (2006) Paternal age and offspring growth: separating the intrinsic quality of young from rearing effects. Behav Ecol Sociobiol 60:672-682

Houston AI, Székely T, McNamara JM (2005) Conflict between parents over care. Trends Ecol Evol 20:33-38

Jourdie V, Moureau B, Bennett ATD, Heeb P (2004) Ultraviolet reflectance by the skin of nestlings. Nature 431:262

Lehtonen PK, Primmer CR, Laaksonen T (2009) Different traits affect gain of extrapair paternity and loss of paternity in the pied flycatcher, Ficedula hypoleuca. Anim Behav 77:1103-1110. doi:10.1016/j.anbehav.2009.01.014 
Lessells CM (1991) The evolution of life histories. In: Krebs JR (ed) Behavioral ecology: an evolutionary approach. Blackwell, Oxford, pp 32-68

Lessells CM (2002) Parentally biased favoritism: why should parents specialize in caring for different offspring? Phil Trans Roy Soc Lond B 357:381-403

Lessells CM (2006) The evolutionary outcome of sexual conflict. Phil Trans Roy Soc Lond B 361:301-317

Lessells CM (2008) Neuroendcrine control of life histories: what do we need to know to understand the evolution of phenotypic plasticity? Phil Trans Roy Soc Lond B 363:1589-1598

Linden M, Møller AP (1989) Cost of reproduction and covariation of life history traits in birds. Trends Ecol Evol 4:367-371

Lundberg A, Alatalo RV (1992) The pied flycatcher. Academic, London

Marshall DJ, Uller T (2007) When is a maternal effect adaptive? Oikos 116:1957-1963

Michl G, Török J, Péczely P, Garamszegi LZ, Schwabl H (2005) Female collared flycatchers adjust yolk testosterone to male age, but not to attractiveness. Behav Ecol 16:383-388

Moreno-Rueda G (2007) Yolk androgen deposition as a female tactic to manipulate paternal contribution. Behav Ecol 18:496-498

Müller W, Groothuis TGG, Eising CM, Dijkstra C (2005) An experimental study on the causes of sex-biased mortality in the black-headed gull - the possible role of testosterone. J Anim Ecol 74:735-741

Müller W, Lessells CM, Korsten P, von Engelhardt N (2007) Manipulative signals in family conflict? On the function of maternal yolk hormones in birds. Am Nat 169:E84-E96

Navara KJ, Hill GE, Mendonca MT (2006) Yolk androgen deposition as a compensatory strategy. Behav Ecol Sociobiol 60:392-398

Pilz KM, Quiroga M, Schwabl H, Adkins-Regan E (2004) European starling chicks benefit from high yolk testosterone levels during a drought year. Horm Behav 46:179-192

Ratikainen II, Kokko H (2010) Differential allocation and reproductive compensation: who deserves the silver spoon? Behav Ecol 21:195-200

Rubolini D, Martinelli R, von Engelhardt N, Romano M, Groothuis TGG, Fasola M, Saino N (2007) Consequences of prenatal androgen exposure for the reproductive performance of female pheasants (Phasianus colchicus). Proc Roy Soc Lond B 274:137-142
Rutkowska J, Cichoń M, Puerta M, Gil D (2005) Negative effects of elevated testosterone on female fecundity in zebra finches. Horm Behav 47:585-591

Ruuskanen S, Laaksonen T (2010) Yolk hormones have sex-specific long-term effects on behavior in the pied flycatcher (Ficedula hypoleuca). Horm Behav 57:119-127

Ruuskanen S, Doligez B, Tschirren B, Pitala N, Gustafsson L, Groothuis TGG, Laaksonen T (2009) Yolk androgens do not appear to mediate sexual conflict over parental investment in the collared flycatcher Ficedula albicollis. Horm Behav 55:514-519

Siitari H, Honkavaara J, Huhta E, Viitala J (2002) Ultraviolet reflection and female mate choice in the pied flycatcher, Ficedula hypoleuca. Anim Behav 63:97-102

Slagsvold T (1997) Brood division in birds in relation to offspring size: sibling rivalry and parental control. Anim Behav 54:13571368

Svensson L (1992) Identification guide to European passerines. Fingraft AB, Sweden

Tobler M, Granbom M, Sandell MI (2007) Maternal androgens in the pied flycatcher: timing of breeding and within-female consistency. Oecol 151:731-740

Trivers RL (1972) Parental investment and sexual selection. In: Campbell B (ed) Sexual selection and the descent of man. Aldine publishing company, Chicago, pp 136-179

Tschirren B, Richner H (2008) Differential effects of yolk hormones on maternal and paternal contribution to parental care. Anim Behav 75:1989-1994

Verboven N, Monaghan P, Evans DM, Schwabl H, Evans E, Whitelaw C, Nager RG (2003) Maternal condition, yolk androgens and offspring performance: a supplemental feeding experiment in the lesser black-backed gull. Proc Roy Soc Lond B 270:2223-2232

Warner DA, Lovern MB, Shine R (2007) Maternal nutrition affects reproductive output and sex allocation in a lizard with environmental sex determination. Proc Roy Soc Lond B 274:883-890

Westneat DF, Sargent RG (1996) Sex and parenting: the effects of sexual conflict and parentage on parental strategies. Trends Ecol Evol 11:87-91

Williams GC (1966) Natural selection, the costs of reproduction, and a refinement of Lack's principle. Am Nat 100:687-690

Wright J, Leonard ML (2002) Evolution of begging: competition, cooperation \& communication. Kluwer, Dordrecht 